\title{
Authente-Kente: Enabling Authentication for Artisanal Economies with Deep Learning
}

\author{
Accepted August 19, 2020 to Al \& Society \\ DOI: 10.13140/RG.2.2.27020.95362/2
}

Corresponding author: Kwame Porter Robinson (kwamepr@umich.edu), Graduate Student, University of Michigan, School of Information, 3360 North Quad, 105 S. State St., Ann Arbor, MI 48109-1285. ORCID\#0000-0003-2663-571X

Co-authors:

Ron Eglash (eglash@umich.edu), Professor, University of Michigan School of Information. ORCID\# 0000-0003-1354-1300

Audrey Bennett, (agbennett@umich.edu), Professor, University of Michigan Penny W. Stamps School of Art and Design. ORCID\# 0000-0002-6763-2622

Sansitha Nandakumar (sansitha@umich.edu), Graduate Student, University of Michigan School of Information. ORCID\# 0000-0001-8651-1415

Lionel Robert (Iprobert@umich.edu), Associate Professor, University of Michigan School of Information. ORCID\# 0000-0002-1410-2601

\begin{abstract}
The economy for artisanal products such as Navajo rugs or Pashmina shawls are often threatened by mass-produced fakes. We propose the use of Al-based authentication as one part of a larger system that would replace extractive economies with generative circulation. In this case study we examine initial experiments towards the development of a cell phone based authentication app for kente cloth in west Africa. We describe the context of weavers and cloth sales; an initial test of a machine learning algorithm for distinguishing between real and fake kente, and an outline of the next stages of development.
\end{abstract}

Keywords: human-machine collaboration; machine learning; artisanal economy; generative justice; industrial symbiosis; ethnocomputing 


\section{Declarations}

\section{Funding}

This research is supported by National Science Foundation (NSF) grant \#1640014 and Mcubed grant \#8330

\section{Conflicts of Interest}

The authors have no conflicts of interest to declare. All co-authors have seen and agree with the contents of the manuscript and there is no financial interest to report.

Availability of data and material

Data used within this work is available at https://github.com/robinsonkwame/kente-clothauthentication

Code Availability

Code used within this work is available at https://github.com/robinsonkwame/oc-svm 


\section{Introduction}

Mass production economies have introduced many ills into social life, including high rates of mental and physical illness from dull repetitive jobs; high rates of environmental degradation; and a "junk food" approach to both physical and informational over-consumption (Michelsen and Bildt, 2003; Coccia 2017; Hunt et al 2018). Artisanal fabrication, in contrast, tends to embody the opposite effect. Artisans often report that they are drawn to their craft because it is an enjoyable and rewarding form of labor. Traditional fabrication methods often use locally sourced and sustainable supply chains. And (at least traditionally) artisanal items were purchased in more thoughtful ways, often establishing a personal relationship between buyer and seller. In our prior work (Eglash et al 2019), we suggested that Al, robotics and other forms of automation, if properly designed and implemented, could gradually scale these beneficial systems towards the development of an artisanal economy. One small step in that direction might be Al guides that help connect consumers with artisanal producers. In this paper we explore a prototype, Authente-Kente, to help guide consumers toward selection of authentic hand-woven kente cloth, and thus diminish income loss due to mass produced fake cloth.

\section{The Problem Context}

Traditional artisanal items often compete with mass-produced fakes. M'Closkey (2010) estimates that out of roughly 2 billion in annual sales of "Native American" goods, about $50 \%$ is not actually of Native origin. Similar problems arise elsewhere: for example, Mehra (2019) reports that due to competition from mass produced fakes, the number of Pashmina shawl hand weavers had dropped from over 100,000 in 2007 to about 10,000 in 2017. The artisanal product of concern in our case study is Kente cloth, a traditional fabric from the West African nation of Ghana. Kente is of great symbolic importance as a symbol of African heritage, not only in Ghana but also abroad. African American students often wear a kente stole at graduation. In January 2018 members of the Congressional Black Caucus wore kente cloth wraps and scarves to the State of the Union Address to protest the rising racism in populist politics (figure 1). 


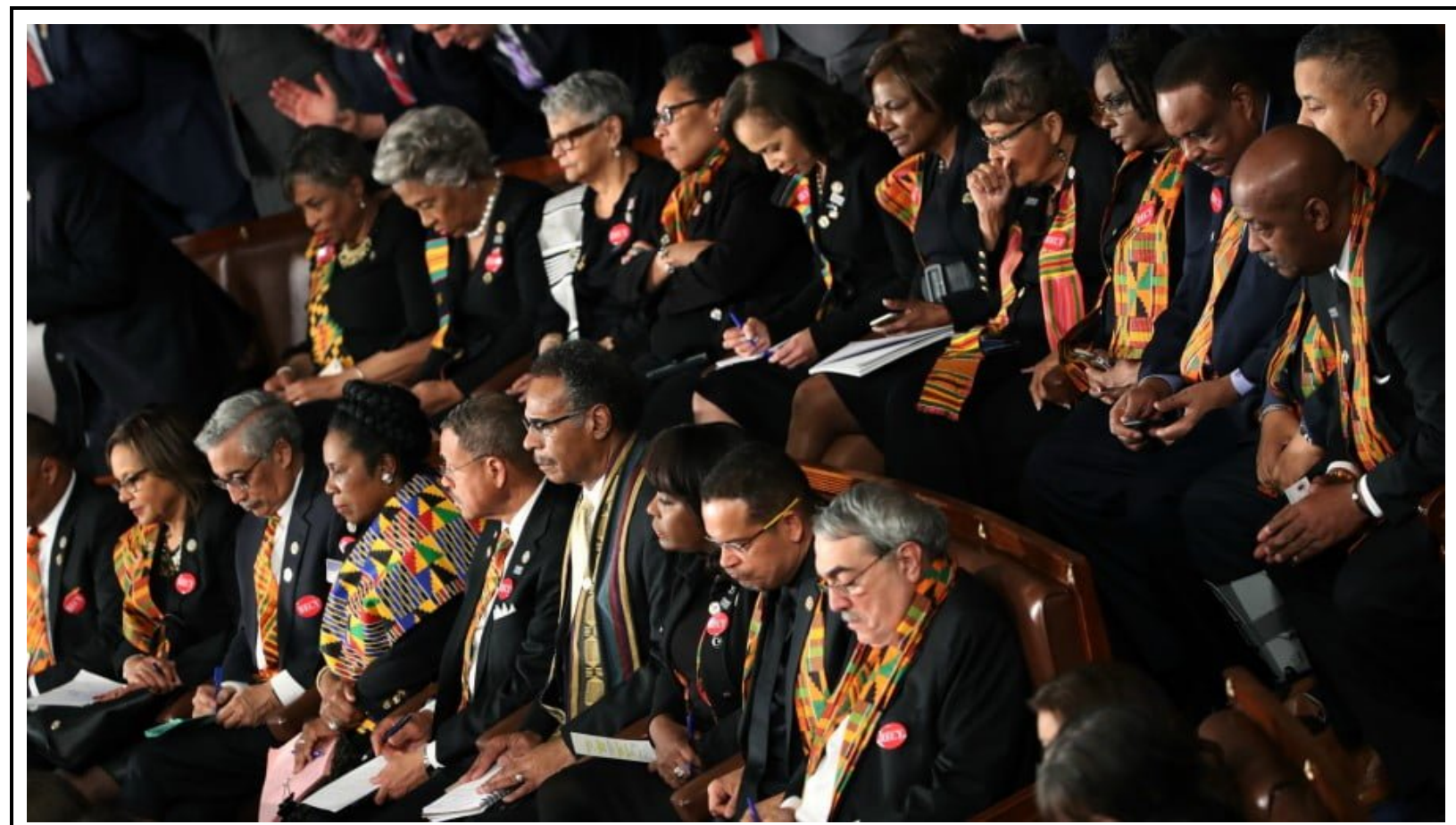

Figure 1: Members of the Congressional Black Caucus wearing kente on Jan 30, 2018.

As in other cases of artisanal production, authentic hand-made kente cloth is in competition with lower cost imported fakes. Howard et al (2016) note the steep decline in Ghana's textile industry due to fake imports: from about 25,000 employed in 1977 to under 3,000 by 2005 . Revenue loss from fake imports of all products (including textiles) is now estimated to be over $\$ 1$ billion in Ghana (Boateng 2019). On the positive side, Ghana's tourism industry contributed $\$ 2.7$ billion, or $6.2 \%$ of the national GDP, in 2017 (Oxford Business Group, 2019). If tourism could be tied to selective purchase of authentic, locally produced textiles, declines for that industry could be reversed. Thus a cell-phone based Al guide for directing tourists towards purchasing authentic textiles seemed like a promising prototype for experimentation in this area.

Prior work on automated authentication of artisanal textiles has not focused on the tourist trade. Rather they are typically made for professional export industries. One exception is the case of Pashmina shawls: the Kashmir government set up a six million dollar testing facility to ensure that the fibers are purely composed from hair of the Pashmina goat (Capra hircus), and issued a microchip-enabled authentication seal (Parvaiz 2017). However these are mainly serving large high-end tourist shops, where shawls are sold for as much as $\$ 1000$ each. Even then, decoding the chip requires an infrared light reader before its code can be checked with an online database. On the whole, the system is somewhat akin to visiting a diamond merchant and asking for authentication of the gem's intrinsic value with respect to provenance and crystalline composition.

On the one hand top-down authentication systems are very helpful in two circumstances. Firstly, in cases of high intrinsic value. Diamonds have a laser-etched serial number if they have been certified (Carl 2016). Individual diamonds are worth thousands or millions. Secondly, for a highly 
regulated industry. Pharmaceuticals may be low cost as individual packets, but the industrial wealth and the power of medical institutions are such that they can afford to maintain and manage a highly regulated system of suppliers and retailers.

Kente cloth weavers, on the other hand are in neither category. Their individual weavings sell for relatively low cost and as a group they are neither regulated nor wealthy. Thus preventing someone from appropriating a barcode meant to be used by weavers and applying it to factory fakes would be very difficult. Indeed one of the best known modern texts on kente cloth is titled "That Copyright Thing Doesn't Work Here" (Boateng 2011).

Kente cloth, in contrast, is generally created by low-income communities in Ghana and sold at relatively low prices. The confusion for tourists comes primarily not from duplicate weavings, but rather kente prints. Mass-produced in factories at very large scales, it replicates the geometric patterns and colors as an image printed on ordinary cloth. We will refer to these as "fake" kente, because from a weaver's perspective it is marketing a phoney version of their craft for lower prices than they can compete with. ${ }^{1}$ What motivates tourists to take efforts to guarantee the authenticity of weaving in the case of Pashmina--the perception of a high intrinsic value due to specific types of fibers and process--is clearly not present in the case of kente prints, at least in the context of large markets where we have observed this confusion first hand.

On the other hand, tourists in the presence of a weaver clearly value the cloth for its hand-made character, even when the fake print version is also available at lower cost. Communities devoted primarily to kente weaving have had some significant success. For example, Edusei and Amoah (2014) examined the labor demographics for the Kwabre East District of Ghana, which includes the villages best known for kente weaving. Out of 27,000 households, an estimated 10,000 jobs were in the textile industry (which includes weavers as well as thread suppliers, tailors, retail vendors, etc.). While we tend to think of tourists visiting a weaver as somewhat artificial or contrived, such personal encounters between maker and buyer are arguably closer to the Indigenous economic tradition than, say, shopping at Ghana's Accra mall (which is modeled on American malls, including food courts, security guards and so on). In the original Indigenous economy, value circulated in the form of collaborative labor groups, rituals of gift exchange, a shared resource commons, and other elements of what is sometimes referred to as "relational economies". Curry and Koczberski (2012), examining how aspects of relational economies still exist today alongside commodity economies, note:

In these transactions, an intrinsic relation exists between the item of exchange and its donor, in that the exchange item can be conceptualised as constituting 'parts of persons' themselves.... From this perspective, the gift contains the embodiment of the donor and is never fully alienated from the person as in commodity transactions.

\footnotetext{
${ }^{1} \mathrm{~A}$ case can be made that the print is not intended to be mistaken for handwoven, and therefore legitimately sold. But we have observed tourists failing to grasp the distinction, so the impact on weavers is the same. There are also distinctions between "authentic prints" made in Ghana, and those produced in foreign countries and smuggled across the border, but that is beyond the scope of this paper.
} 
This helps us see what is happening when hand-woven kente is selected by tourists in areas where they see it made, in contrast to the commodity transaction with cheaper kente prints in places like Accra's National Center for Culture, which is Ghana's largest tourist market. Unlike Pashmina, which can claim value in the material properties (as a commodity), exporting handmade kente to sites of mass consumption like a mall breaks the relational link that was (and is) a crucial source of its value. Thus it makes sense to seek an authentication process that can operate in more generative modes. To merely distinguish between real and fake might be appropriate for commodity marketing, but if $\mathrm{Al}$ is to play a role in the transition to a generative economy, it needs to facilitate a richer set of producer/consumer relationships.

If we think about the commodity-based, mass manufacturing system as something that has broken relationships, then a system for more generative forms of authentication might be understood metaphorically as a kind of prosthetic for replacing missing parts and restoring functionality to the social organism. To this end we envision a system in which artisans can use cell phones (ubiquitous in Ghana) to upload making-in-action videos, conversations with elders, comments on favorite weaving patterns, symbolic meanings of colors and shapes, or other media representations that tell their story from the production side. From the buyer side, automated identification of a textile would enable locating a best guess for its point of origin, access to the producer's media (including identification of the pattern's symbolic meanings), and perhaps even opportunities for two way communication ("please be a guest speaker in my class", "my wife wants to show you the beadwork she sells", etc.). And there is no reason this must be restricted to textiles; any purchase could be approached in this way, allowing more insightful and purposeful buying and selling.

Setting aside for the moment this ambitious vision of what generative authentication might become, a basic functionality for distinguishing real handwoven versus fake printed patterns seemed like the most fundamental first step. Below we describe our initial experiments using an Authente-Kente task specific machine learning pipeline to make this basic distinction.

\section{Overview}

In selecting the Al method, we began with the observation that the problem of distinguishing between authentic handwoven and fake (printed) kente patterns is broadly related to anomaly or fraud detection (Hodge and Austin 2004). There are many possible approaches to automated anomaly detection (Das 2009); we narrowed our choice by taking into account two constraints.

First, we can expect the proportion of authentic to fake instances will vary greatly by site. Typically, tourists would encounter far more instances of printed kente in the Accra mall than would in the village of Asonomaso, within the Kwabre East District, where authentic kente is ubiquitous. In other cases the ratio may be closer to $1: 1$. Thus it is important that AuthenteKente perform well against both authentic and fake instances of kente cloth, independent of authenticity prevalence. This raises an unusual challenge in that most statistical anomaly derivations assume a fixed ratio of anomalous/normal cases derived from a static application domain (Das 2009). Our framework for generative authentication, in contrast, requires that 
solutions work across a variety of contexts with varying and unknown ratios of anomalous cases. Thus we began prioritizing success metrics by assuming equal prevalence, keeping in mind that re-assessing this assumption might be needed as data comes in from real-world deployment.

Second, as a practical matter we had a limited number of authentic kente cloths available as samples. Extensive field work might slowly accumulate more, but nothing like the thousands of cases required if we wanted to use whole cloths as the samples. The relatively high number of sample cases is typical in machine learning, particularly Deep Learning (LeCun, Bengio, and Hinton 2015), where convolutional neural networks (CNNs) have facilitated empirical success for complex visual recognition tasks. Some strategies have reduced the training set size: in Yadav and Jadhav (2019) for example, automated medical image classification for rare disease diagnosis out-performed trained doctors, using only 5,232 training images. However that is still magnitudes of order greater than our sample size of a dozen or so cloths. To resolve this issue we created many local samples of each cloth; we refer to these as "swatches." This was facilitated by the visual complexity of kente, which has a wide variety of geometric design elements, grouped in particular arrangements, and organized in arrangement patterns. We followed exactly the same process with fake whole cloths, deriving sample swatches from them in the same experimentally controlled manner. Finally, we note that in a real-world situation, tourists will likely find it easier to casually take cell phone photos of folded cloth (swatches) than ask that each cloth be fully unfolded and extended.

\section{Pipeline Overview}

As noted above, our initial prototype addresses the problem of guiding a tourist towards authentic hand-woven kente cloth as a socio-technical decision problem to be supported by an efficient machine learning pipeline. The problem domain defined by this envisioned future application, illustrated in Figure 2, shows the user taking a close up photo of a whole kente cloth--i.e. a swatch--and getting back an authentic/fake classification from the application. In our laboratory mockup, we begin with a whole cloth, which is broken into swatch images (artificially creating what real-world users would do) and provide the swatch to the machine learning pipeline. Training on swatch samples for both authentic and fake cloths then produces a reliable classification system using a CNN. We experimentally investigated the success of the pipeline on randomly generated swatch images as described in later sections. 


\section{Authente-Kente}

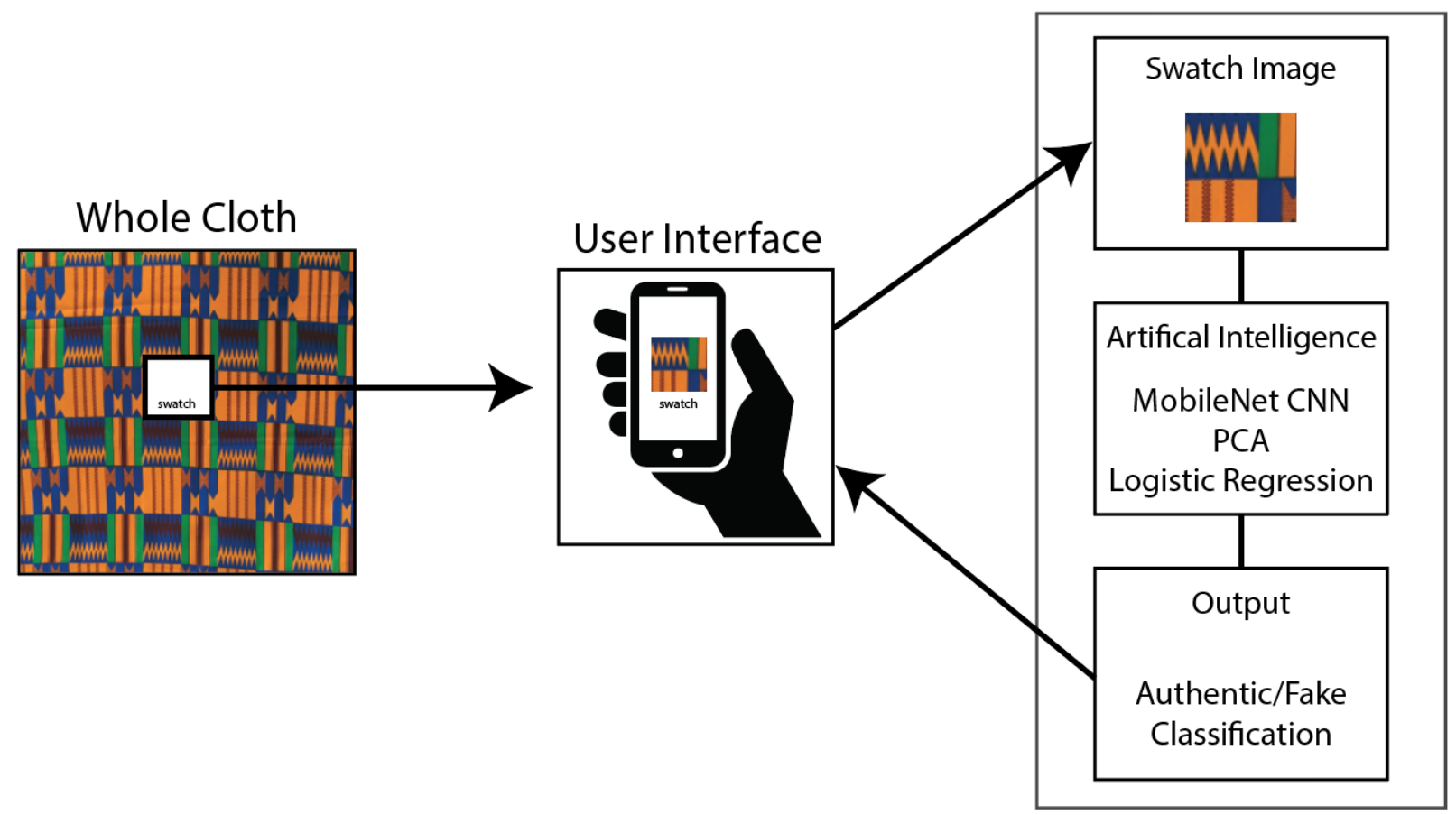

Figure 2: Current Experimental Problem Domain

\section{Whole Cloth Validity}

We generated swatches from 16 whole kente cloth samples, given in Figure 3: eight authentic, and eight fake (i.e. machine printed), and represented in Figure 4: sample grid and tonal color distribution of fake and authentic swatches. Authentic cloths included 7 museum pieces, and one collected by one of the authors in the village of Bonwire in 2011. The fake samples were sourced from a variety of manufacturers. The typical whole cloth sample was imaged at least $2000 \times 2000$ pixels, with the smallest sample spanning $633 \times 633$ pixels (museum, authentic) and the largest sample spanning $2981 \times 2981$ pixels (in-hand, fake). Environmental lighting conditions for fake whole cloth photos were kept similar to that of the authentic whole cloths through omnidirectional lighting without occlusions. All whole cloth samples were square. Table 1 contains the whole cloth specifications. 

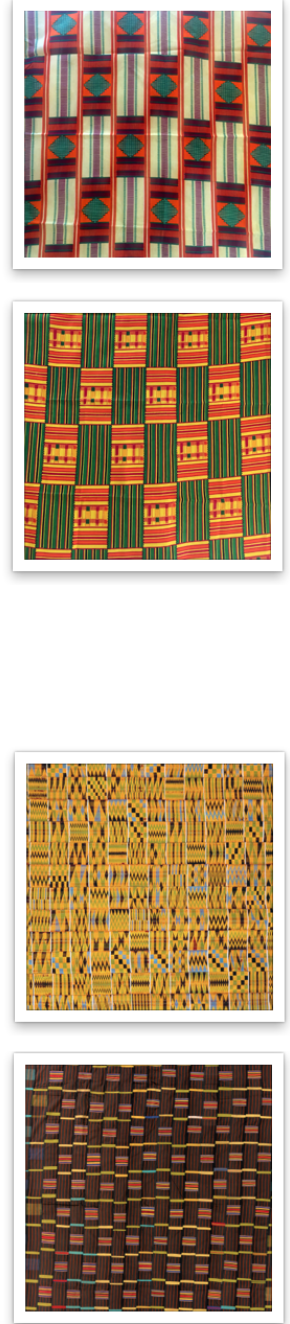
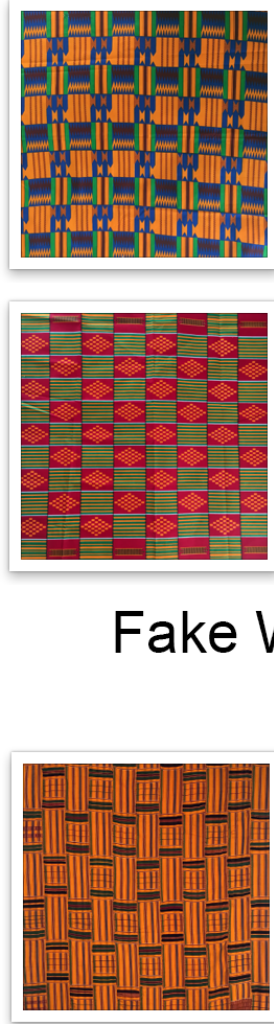

Fake Whole Cloths

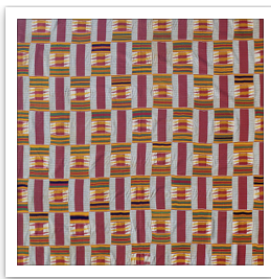

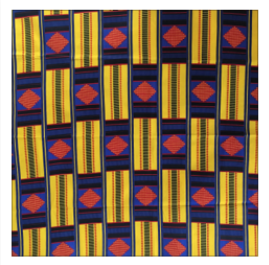
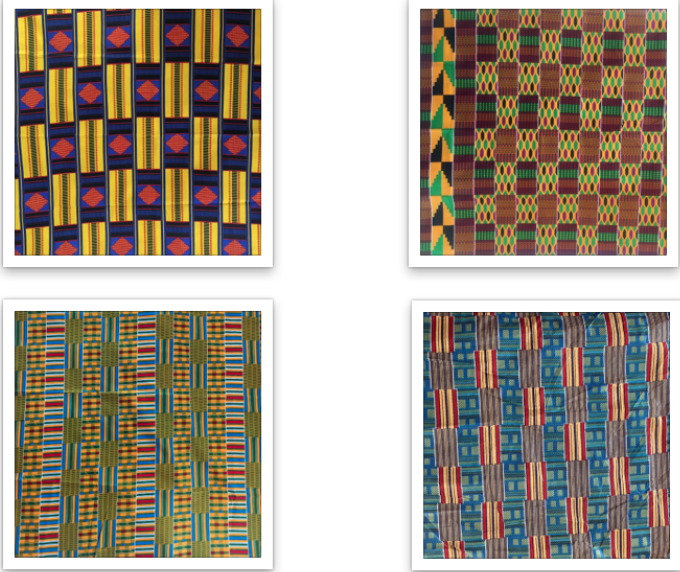

\section{Authentic Whole Cloths}

Figure 3: Eight whole kente cloth samples in each group (fake and authentic)

\begin{tabular}{|c|c|c|c|c|c|c|c|}
\hline \multicolumn{2}{|c|}{} & \multicolumn{3}{|c|}{ Environmental Conditions } & \multicolumn{3}{c|}{ Sample Size } \\
\hline Source & Type & Lighting & Occlusion & $\begin{array}{c}\text { Dates \& } \\
\text { Times of } \\
\text { Day }\end{array}$ & $\begin{array}{c}\text { Whole } \\
\text { Cloths }\end{array}$ & Swatches & By Type \\
\hline Museum & Authentic & Omni* & None & Varied & 7 & 875 & 1000 \\
\hline In-hand & Authentic & Omni $^{*}$ & None & $\begin{array}{l}1 / 23 / 2020 @ \\
\sim 5 p m\end{array}$ & 1 & 125 & \\
\hline
\end{tabular}




\begin{tabular}{|l|c|c|c|c|c|c|c|}
\hline & Fake & Omni* & None & $\begin{array}{c}5 / 1,5 / 15 \& \\
6 / 182020 @ \\
\sim 5 \mathrm{pm}\end{array}$ & 8 & 1000 & 1000 \\
\hline
\end{tabular}

Table 1: Sample environmental condition and sizes

* Omni refers to omnidirectional natural lighting without lighting hotspots

\section{Swatch Construct Validity}

Figure 4.A and Figure 4.B radially lay out ${ }^{2}$ swatches in a mosaic from lighter to darker tones without replacement starting from the center. In Figure 4.C the red, green and blue (RGB) swatch tonal histogram of both authentic and fake swatches share similar shapes although the fake swatch color is more peaked at the mid-dark tones. Taking a standard model of human color perception into account, in Figure 4.D, the $\mathrm{LAB}^{3}$ histograms also indicate similar shapes. The $L A B$ differences include a peak in $L^{*}$ (lightness) values for fake swatches as compared to authentic swatches, wider $a^{*}$ (green/red) distribution in fake swatches and a sharper $b^{*}$ (blue/yellow) distribution in authentic swatches. In Figure 4.E, as a simplified cylindrical volume, fake and authentic swatches are virtually the same. Only the center of mass differs and mainly along the $L^{*}$ component.

In aggregate, these differences are visually identifiable as in Figure 4.A and Figure 4.B as authentic swatches having less "saturation" relative to the artificial inks of fake swatches and that fake swatches have darker inks that are somewhat darker than authentic swatches. In addition, we can see that the range in 4.A is relatively smooth, while that of $4 . B$ changes by discrete steps. Adherence to traditional patterns (4.B) versus introduction of invented patterns (4.A) may be causing this effect. Another possible cause is that weavers are purchasing thread from similar supply chains, and these threads tend to be offered in relatively discrete categories (the 6 primary and secondary colors). In contrast textile inks are marketed to manufacturers in a continuous range of colors. We have endeavored to ensure that these color range differences are not accidentally added by lighting, camera angle or other external factors, as noted in our Whole Cloth Validity section above.

The dissimilarity of Figure 4.A and Figure 4.B may give the impression that it will be easy to identify a particular swatch as in either the fake or authentic category. But these are showing us the aggregate characteristics. Any single swatch, as a user would examine in a market, is a single realization of color variables drawn from largely overlapping distributions. Viewed statistically, the overlapping distributions induce similar mean and variance statistics that prevent $L A B$ components and tone from being discriminative enough to achieve high classification accuracy. This discriminative difficulty is mirrored by our original problem

\footnotetext{
${ }^{2}$ Using a mosaic layout algorithm by $d v d t h o$ available at https://github.com/dvdtho/python-photo-mosaic

${ }^{3}$ The CIE L*a*b* color space model, abbreviated as LAB, is a non-linear model based on human color perception and expresses color in terms of $L^{*}$, lightness, from black (0) to white (100), $a^{*}$ from green to red and $\mathrm{b}^{*}$ from blue to yellow.
} 
motivation that tourists, using color alone, are not reliably able to determine if a cloth is authentic or fake. This suggests that a more powerful model is needed.

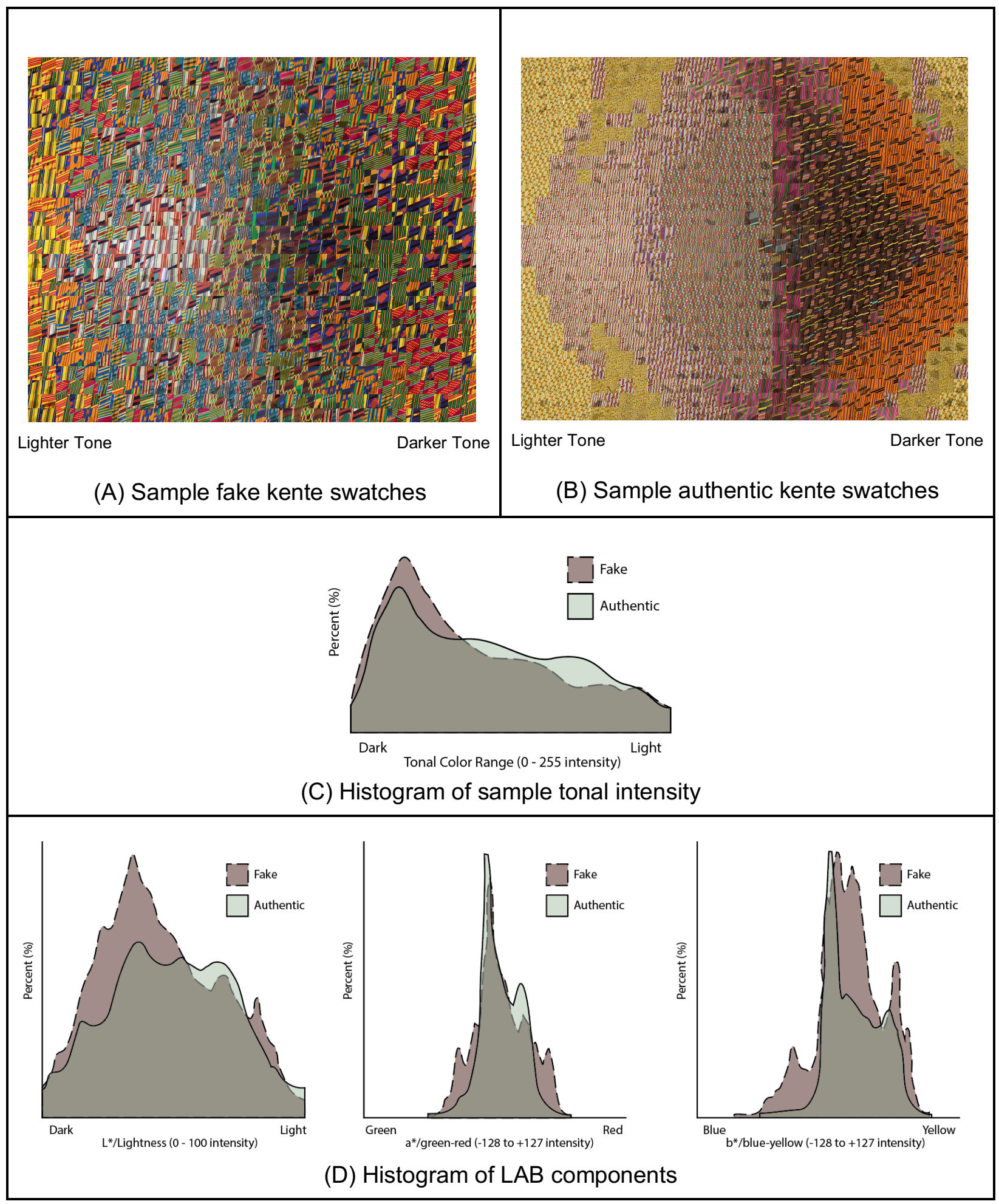




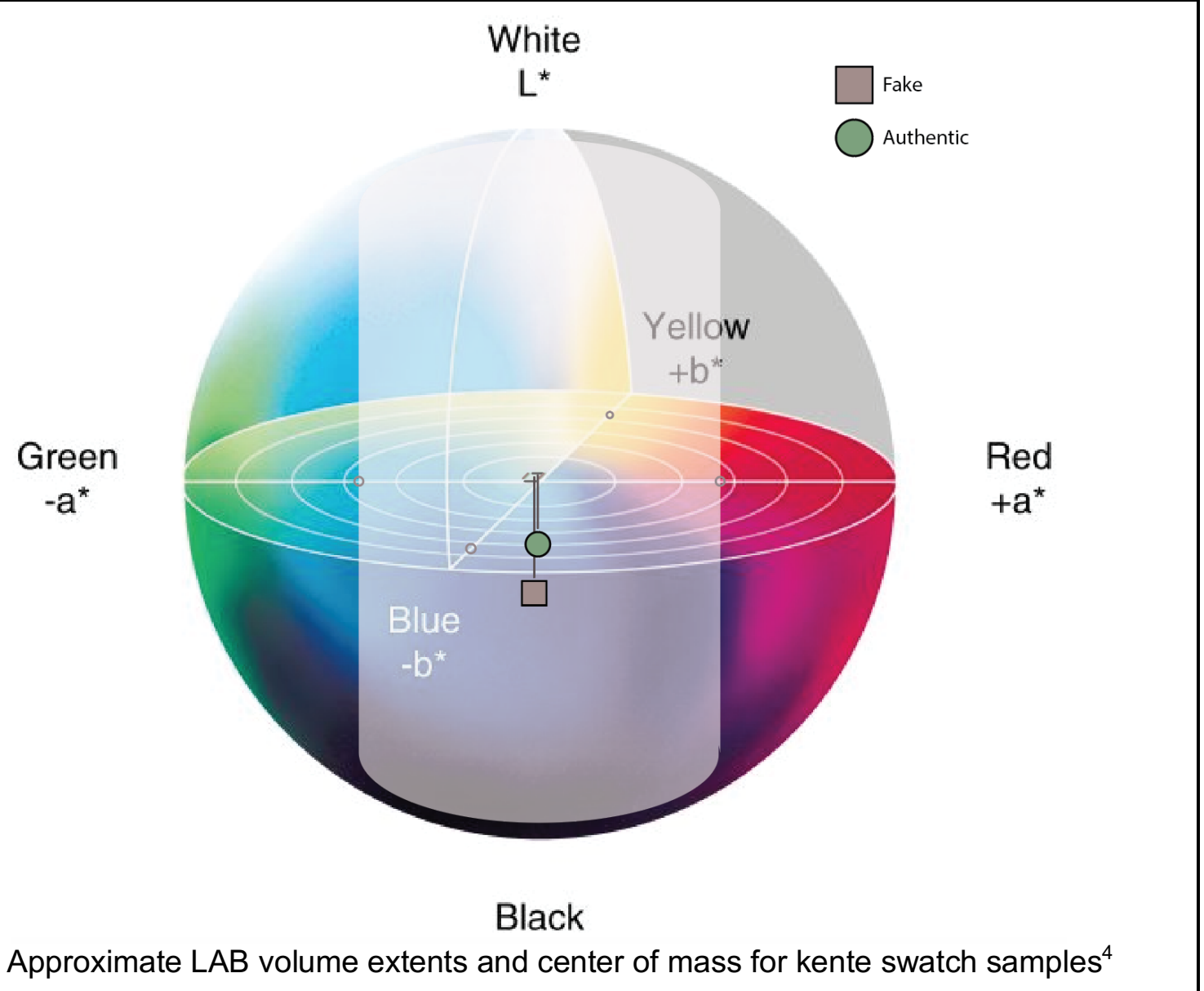

Figure 4: Sample grid, tonal color, LAB color histograms, sphere of fake and authentic swatches

In addition to the challenge of making the fake/authentic distinction from these sets of swatches, we can expect that tourists casually taking images will introduce many sources of natural variation. Data augmentation (Shorten and Khoshgoftaar 2019) provides a controlled method for post-processing to be consistent with sources of natural variation. We endeavored to replicate the natural image variation through data augmentation that randomly applies minor image transformations to each of the 2,000 swatches. These mimicked the real-world variability in the angle of the photo, the type of phone, and the environmental lighting.

Unless the user's wrist holds the phone perpendicularly the resulting swatch will be oblique to the camera lens. Therefore we randomly rotate a swatch along three dimensions (the axes $\theta, \phi$, $\gamma$ ). The rotation of the plane formed by axes $\theta$ and $\phi$ simulate rotation parallel to the kente cloth and the rotation along the axis $\gamma$ simulates cell phone camera forward rotation formed by the natural tit of the wrist. The image sensor in the phone has a unique color profile such that swatch color can subtly differ across phones. We randomly and slightly perturb the red, green and blue color channels of the swatch image to induce variations in color. Finally, the user

\footnotetext{
${ }^{4}$ Photo Reference Credit "About Color Management," Sony. 2020. Accessed: https://www.sony.co.uk/electronics/support/about-color-management
} 
location creates the possibility of shadows falling on the swatch. Each swatch image has a randomly projected shadow on it ranging intensity from $0 \%$ (invisible) to $10 \%$ in lightness. Source code for generating this dataset from scratch ${ }^{5}$ is openly available. We account for these expected sources of natural visual variation through data augmentation. Figure 4 lays out swatches from lighter to darker. The red, green and blue (RGB) swatch color distribution of both authentic and fake swatches share similar shapes although the fake swatch color is more saturated (e.g. vivid). That the tonal color distributions largely overlap another means that color (including hue, lightness and saturation) alone is not discriminative enough to distinguish between authentic and fake kente.

\section{Dataset Generation}

We took care to create a balanced dataset of authentic and fake kente swatches. From each whole cloth 125 swatches are generated, per Swatch Construct Validity. Swatches of size 224 by 224 pixels are randomly extracted from whole cloth samples to create a balanced dataset of 1000 authentic and 1000 fake swatches. The data are split into training (750 cases), validation (750 cases) and evaluation (500 cases) datasets. Experimental Data Evaluation discusses the partitioning of the dataset in additional detail.

\section{A Brief Background on Convolutional Neural Networks (CNNs)}

Progress on ImageNet (Russakovsky et al. 2014), a classic computer vision object recognition challenge involving thousands of objects in natural settings, had largely stalled in the field of computer vision in early 2010. However, convolutional neural networks (CNNs), first used by Alex Krizhevsky et al. (2012), showed the potential to dramatically improve state of the art performance (in their case on the ImageNet Object recognition task). CNNs are a type of deep learning modelled after the human visual cortex and achieve their high performance by mapping pixels to hidden neuron layers. The hidden layers provide a variety of convolutions (subsampling processes to generate a set of data driven features). Finally, it correlates those features to class probabilities (e.g. classification decisions) within the final layer. Massive numbers of samples and stochastic gradient descent are techniques required for efficient learning of what features correlate to class probabilities in deep learning, including CNNs.

Using an already trained CNN, one can take advantage of the initial layers, which have already learned to extract visual features such as edges and textures, and limit training for the particular case at hand to its task-specific needs. One advantage of that is reducing training time; another is environmental. Typically, hardware acceleration in the form of graphical processing units (GPUs) are utilized, and GPUs require significant amounts of power while running the backpropagation neural network training process over many iterations (Strubell, Ganesh, and Mccallum 2019). We therefore used an already trained CNN, MobileNet v2 (Howard et al. 2017), designed for efficient real time prediction within mobile phones.

${ }^{5}$ See https://github.com/robinsonkwame/kente-cloth-authentication 
Specifically, we carry out an indirect form of transfer learning by using the MobileNet object classification likelihoods as a feature vector passed to the next stage of Authente-Kente, principal component analysis. The feature vector is the output layer of the MobileNet CNN and has $1280 * 7^{*} 7=62,627$ feature vector variables that take on particular values (e.g. predicted object likelihoods) for a given $224 \times 224$ pixel subsection presented at the input layer. We discuss how the feature vector is reduced in the following section.

\section{Dimensionality Reduction}

Given that we only have 2,000 kente swatches the feature vector size exceeds the number samples and presents an ill-posed statistical learning problem (e.g. $p>>n$ ) that requires a reduction of the feature vector size. We apply the standard practice of principal component analysis (PCA), a statistical technique that orthogonally transforms a larger matrix into a smaller matrix of linearly uncorrelated components called principal components. In our case we have 1,500 samples across the training and validation dataset (see Experimental Data Evaluation). We opt to reduce the feature vector to a standard $n / 2$ principal components that together explain $77 \%$ of the total variance. The component eigenvalues are all larger than 1 so we retain them as is standard. The PCA decomposition explains a large percentage of the input feature vector variance while reducing the input matrix to dimensions that present a well posed statistical problem.

\section{Logistic Regression}

Logistic regression is a common statistical model for binary outcomes and is used to model the decision of whether a subsection is from fake or authentic kente cloth (e.g. -1 for fake kente, 1 for authentic kente). In our formulation we use the PCA decomposition of the MobileNet feature vector as independent variables from which to learn a logistic regression model. Regression is particularly applicable in an applied setting because it is computationally efficient to evaluate. Regression models are learned by statistically fitting model coefficients through an optimization process that uses a loss function to numerically express the difference between predicted outcomes and actual outcomes (e.g. error). Domain specific loss functions often better generalize fitted model coefficients by controlling for known sources of error in the problem domain.

Authentication error can be characterized by the cost and type of misclassification. A type of kente may be misclassified as a false positive or a false negative, with related statistical measures sensitivity and specificity. In a random marketplace the prevalence of fraud is unknown and so we assume an equal cost of misclassification to avoid bias. To control for equal misclassification cost and type we use the F1-macro loss function to induce a logistic regression model that equally minimizes and balances error. Here we assume that authentic and fake kente are equally likely and their misclassification equally costly.

\section{Experimental Data Evaluation}

Experimental evaluation in machine learning situations is complicated by the difficulty of estimating the standard error of a model. The standard approach is to use cross validation to 
estimate appropriate model parameters and performance, using a training dataset for training the machine learning model and using a separate dataset, the validation dataset, to validate chosen model parameters. A third, separate dataset of unseen whole cloths, the evaluation dataset, is used to estimate the out of sample model error (Kohavi 1995). To maximize external and internal validity we separate the data into training (750 cases), validation (750 cases), and evaluation (500 cases) datasets. The training and validation swatch datasets are generated from the same whole cloth images. The evaluation swatch dataset is generated from a different set of whole cloth images; no swatch in the evaluation dataset has been seen by the proposed model derived from the training and validation datasets. Source code for generating our dataset from scratch and running the machine learning experiment ${ }^{6}$ is openly available.

To claim significance we evaluate our pipeline along a gamut of standard binary classification metrics: precision, recall, macro-F1 and weighted-F1 and accuracy (Tharwat 2018). We use precision, recall, macro-F1 and weighted-F1 to support our claims of significance and set purposively low thresholds a priori as given in Table 2 since the problem of visual kente authentication has not been addressed in the literature. We answer the experimental question of whether it is possible to significantly and efficiently decide if a swatch belongs to an authentic or fake kente whole cloth. This is done through a computer science experiment involving training, validation and evaluation datasets, as detailed in Table 3. Computational efficiency is supported by using a pretrained MobileNet (Howard et al. 2017) model, a CNN specifically designed for use on cell phones, and logistic regression as our decision algorithm, which reduces to matrix multiplication with coefficients. Several efficient algorithms for PCA are commonly available.

\begin{tabular}{|c|c|}
\hline A Priori Metric & A Priori Threshold \\
\hline Per Class Precision & 0.60 \\
\hline Per Class Recall & 0.60 \\
\hline Macro F1 & 0.60 \\
\hline Weighted F1 & 0.60 \\
\hline
\end{tabular}

Table 2: Threshold criteria for establishing significance

\begin{tabular}{|c|c|c|c|}
\hline \multicolumn{2}{|c|}{ Dataset } & \\
\hline Unseen Cases & \multicolumn{2}{|c|}{ Shared Cases } & Experimental Step \\
\hline Evaluation & Validation & Training & \\
\hline
\end{tabular}

${ }^{6}$ See https://github.com/robinsonkwame/oc-svm 


\begin{tabular}{|c|c|c|c|}
\hline & & Fit PCA on Training & 1 Run PCA(375 dim) \\
\hline \multirow[t]{5}{*}{$\mathrm{PCA}($ Eval $) \rightarrow$ Eval $_{\mathrm{PCA}}$} & $\mathrm{PCA}(\mathrm{Val}) \rightarrow \mathrm{Val}_{\mathrm{PCA}}$ & $\mathrm{PCA}($ Train $) \rightarrow$ Train $_{\mathrm{PCA}}$ & 2 Transform datasets \\
\hline & & $\begin{array}{c}\left.\text { CrossValidation(Train }{ }_{\mathrm{PCA}}, \mathrm{LR}\right) \rightarrow \\
L R_{\text {train }}\end{array}$ & $\begin{array}{l}3 \text { Train, cross-validate } \\
\text { Logistic Regression }\end{array}$ \\
\hline & \multicolumn{2}{|c|}{$L R_{\text {train }}\left(\right.$ Val $\left._{\mathrm{PCA}}\right) \rightarrow$ Pred $_{\mathrm{Val}}$} & $\begin{array}{l}4 \text { Predict against } \\
\text { validation swatches }\end{array}$ \\
\hline & \multicolumn{2}{|c|}{ Significance $\left(\right.$ Pred $\left._{\mathrm{Val}}\right)>$ A Priori Thresholds } & $\begin{array}{l}5 \text { Examine significance } \\
\text { metrics, proceed }\end{array}$ \\
\hline & \multicolumn{2}{|c|}{ CrossValidation(Val ${ }_{\mathrm{PCA}}+$ Train $\left._{\mathrm{PCA}}, \mathrm{LR}\right) \rightarrow L R_{\text {train+val }}$} & $\begin{array}{l}6 \text { Train, cross-validate } \\
\text { Logistic Regression on } \\
\text { all shared cases }\end{array}$ \\
\hline \multicolumn{3}{|c|}{$L R_{\text {train }+v a l}\left(\right.$ Eval $\left._{\mathrm{PCA}}\right) \rightarrow$ Pred $_{\text {Eval }}$} & $\begin{array}{l}7 \text { Predict against } \\
\text { evaluation swatches } \\
\text { (never before seen } \\
\text { cases) }\end{array}$ \\
\hline \multicolumn{3}{|c|}{ Significance $\left(\right.$ Pred $\left._{\text {Eval }}\right)>$ A Priori Thresholds } & $\begin{array}{l}8 \text { Examine significance } \\
\text { metrics, report any } \\
\text { results exceeding } \\
\text { thresholds }\end{array}$ \\
\hline
\end{tabular}

Table 3: Initial Authente-Kente Experimental Steps

\section{Results}

Following the experimental setup given in Table 3, a logistic regression model was first cross validated against the training dataset and results were observed to exceed the a priori threshold. Then those parameters were used to re-train the same logistic regression model on the combined training and validation dataset. The re-trained model was then evaluated on the evaluation dataset, as indicated in step 7 of Table 3 and the resulting predictions scored as indicated in step 8 with all metrics exceeding the a priori threshold. A summary of results is given in Table 4. The training and validation datasets had 750 cases each and the evaluation dataset had 500 cases. The number of true cases per class is indicated in the support column. There are 250 fake and authentic cases within the evaluation dataset.

\begin{tabular}{|r|c|c|c|c|}
\hline \multirow{2}{*}{ Evaluation Dataset } & \multicolumn{4}{|c|}{ Metric } \\
\hline Class & Precision & Recall & F1 Score & Support \\
\hline Fake & 0.88 & 0.88 & 0.88 & 250 \\
\hline Authentic & 0.88 & 0.88 & 0.88 & 250 \\
\hline Accuracy & Precision & Recall & F1 Score & Support \\
\hline
\end{tabular}




\begin{tabular}{|r|c|c|c|c|}
\hline Macro Average & 0.88 & 0.88 & 0.88 & 500 \\
\hline Weighted Average & 0.88 & 0.88 & 0.88 & 500 \\
\hline
\end{tabular}

Table 4: Summary of Authente-Kente machine learning pipeline experimental results

* all metrics exceed a priori threshold values

Relative to the a priori metrics given in Table 2 the Authente-Kente machine learning pipeline exceeds the thresholds by large margins. Noticeably, a large majority (recall of $88 \%$ ) of authentic swatch cases are identified and when authentic cases are identified they are accurately predicted to be authentic (precision of $88 \%$ ). This balance between recall and precision is reflected in both macro and weighted averages of $88 \%$. It is important to note that the precision and recall scores are the same. Per the Logistic Regression section, the loss function induces a learned model trained to equally minimize across error types. Precision and recall can be the same when the number of swatches that were actually authentic, false negatives, are the same as the number of swatches that are actually fake, false positives, as observed in Table 4. This is because precision and recall only differ in their denominator, using false positives and false negatives, respectively.

\section{Discussion \& Future Work}

These results against a large number of unseen kente cloth swatches suggest that our implementation significantly captures part of the visual distinction between authentic and fake kente cloth. Although the results are significant, in future work the application needs to be connected to its larger social-technical context. One important social context is the local prevalence of fake kente where the application is in use. As an example, imagine 500 whole kente with a $5 \%$ counterfeit rate. In this case there are 25 fake whole cloths. In this low prevalence example, with a recall of 0.88 Authente-Kente would incorrectly identify 60 authentic whole kente as false. If the user received the authentication decision "likely fake" there is only a $29 \%$ chance that it was accurate in this example. An application that was connected to local artisans and regional data could continue to train as a way to improve accuracy, or selectively account for prevalence to improve overall decision reliability. By design the application makes no assumption and assumes the prevalence rate is equal. This can be addressed in future work with the mobile application that contains the machine learning pipeline.

The components of this machine learning pipeline are computationally efficient and we expect a real world solution to run in real-time. The nearly $1 / 3$ improvement over the a priori threshold provides an indication of experimental significance to the user and the possibilities for becoming one element in a larger platform connecting buyers and artisans. Another element for such platforms might be in STEM education, "whiteboxing" the system to provide an example for how Al can be of social benefit. Our prior work in Ghana shows statistically significant improvement in STEM lessons anchored in this kind of merger between computing and culture (Babbitt et al 2015; for access to the culture-based learning software see https://csdt.org/).

A third element might be kente pattern identification. There is a wealth of symbolic meanings, histories, aphorisms and other details woven into the cloths, but easily forgotten (if they were 
communicated at all). Adding an Al application connecting, for example, a video clip of an elder explaining the meaning of a particular symbol, pattern or style would give such a platform valueadded significance. Further extensions could be obtained by ensuring the sustainability of raw material sources. We have explored this in our work on Ghanaian adinkra textiles (https://generativejustice.org/solar-dye-in-ghana/), and it could be further enhanced using GIS, loT and other systems.

A final element for consideration is enabling the sharing and collection of video recordings of textiles being created by artisans. The ability to connect to someone who creates the kinds of kente cloth and patterns just purchased creates a "value added" that benefits both consumer and producer. In future work, our envisioned system will enable partners in Ghana to directly collect images of kente cloth and patterns. Generally, Al and associated ICT could be utilized as a means to create an ecosystem of services that enhance unalienated labor, sustainable feedstocks and the expressive values of cultures past and future, as we have outlined elsewhere (Eglash et al. 2019).

\section{References}

Babbitt, W., Lachney, M., Bulley, E., \& Eglash, R. (2015). Adinkra mathematics: A study of ethnocomputing in Ghana. Multidisciplinary Journal of Educational Research, 5(2), 110-135.

Boateng, D. Ghana loses over GHC 5.7 billion to fake and counterfeit goods. Panavest, May 20 2019. Accessed online 2/23/2020 from https://www.panavest.com/ghana-loses-over-ghc-5-7billion-to-fake-and-counterfeit-goods/.

Coccia, M. (2017). Global Negative Effects of the Technological Change on Human Health: The High Incidence of Cancers in the Anthropocene.

Curry, G. N., \& Koczberski, G. (2012). Relational economies, social embeddedness and valuing labour in agrarian change: An example from the developing world. Geographical Research, 50(4), 377-392.

Das, K. (2009). Detecting patterns of anomalies (No. CMU-ML-09-101). CMU Pittsburgh PA Machine Learning Department.

Edusei, J., \& Amoah, P. A. (2014). Cultural Tourism and Employment Creation Nexus: Evidence from Kente Weaving and Wood Carving Industries in Kwabre East District, Ghana. Journal of Economics and Sustainable Development, 5(8).

Eglash, R., Robert, L., Bennett, A., Robinson, K. P., Lachney, M., \& Babbitt, W. (2019).

Automation for the artisanal economy: enhancing the economic and environmental sustainability of crafting professions with human-machine collaboration. AI \& SOCIETY, 1-15. 
Hodge, Victoria J., and Jim Austin. 2004. "A Survey of Outlier Detection Methodologies." Artificial Intelligence Review 22 (2): 85-126. https://doi.org/10.1007/s10462-004-4304-y.

Howard, Andrew G., Menglong Zhu, Bo Chen, Dmitry Kalenichenko, Weijun Wang, Tobias Weyand, Marco Andreetto, and Hartwig Adam. 2017. "MobileNets: Efficient Convolutional Neural Networks for Mobile Vision Applications." ArXiv:1704.04861 [Cs], April. http://arxiv.org/abs/1704.04861.

Howard, E., Aboagye, I., \& Quarcoo, J. (2016). Causes and Effects of the Dwindled State of the Ghana Textile Industry. International Journal of Advanced Scientific Research \& Development, 3(4), pp 1-12.

Kohavi, Ron. 1995. "A Study of Cross-Validation and Bootstrap for Accuracy Estimation and Model Selection," August, 7.

Krizhevsky, Alex, Ilya Sutskever, and Geoffrey E Hinton. 2012. "ImageNet Classification with Deep Convolutional Neural Networks." In Advances in Neural Information Processing Systems 25, edited by F. Pereira, C. J. C. Burges, L. Bottou, and K. Q. Weinberger, 1097-1105. Curran Associates, Inc. http://papers.nips.cc/paper/4824imagenet-classification-with-deep-convolutional-neural-networks.pdf.

LeCun, Bengio, and Hinton. 2015. "Deep Learning." Nature 521 (May). https://www.nature.com/articles/nature14539.

Melissa G. Hunt, Rachel Marx, Courtney Lipson, and Jordyn Young (2018). No More FOMO: Limiting Social Media Decreases Loneliness and Depression. Journal of Social and Clinical Psychology: Vol. 37, No. 10, pp. 751-768.

https://doi.org/10.1521/jscp.2018.37.10.751

M'Closkey, K. (2010). Novica, Navajo Knock-Offs, and the 'Net. In Moberg, M. (Ed), Fair trade and social justice: Global ethnographies. NYU Press.

Mehra, P. (2019). The rise of the machines: Kashmir's weavers fear for their future. Al Jeerza, July 19. Accessed 2/22/2020 from https://www.aljazeera.com/ajimpact/rise-machines-kashmirweavers-fear-future-190718043049255.html.

Michelsen, H., \& Bildt, C. (2003). Psychosocial conditions on and off the job and psychological ill health: depressive symptoms, impaired psychological wellbeing, heavy consumption of alcohol. Occupational and environmental medicine, 60(7), 489-496.

Oxford Business Group (2019). Ghanaian tourism grows with increased visitors and new funding strategies. https://oxfordbusinessgroup.com/overview/renewed-ambitions-influx-foreigntourists-and-priority-funding-strategies-are-driving-growth-sector.

Parvaiz, A. (2017). How a nanotechnology based microchip can help you identify real Pashmina https://factordaily.com/pashmina-certification-authenticity-nanotechnology-kashmir/. 
Strubell, Ganesh, and Mccallum. 2019. "Energy and Policy Considerations for Deep Learning in NLP." ACL 2019, June.

https://www.researchgate.net/publication/335778882 Energy and Policy Consideration s for Deep Learning in NLP.

Tharwat, Alaa. 2018. "Classification Assessment Methods." Applied Computing and Informatics, August. https://doi.org/10.1016/j.aci.2018.08.003.

Yadav, Samir S., and Shivajirao M. Jadhav. 2019. "Deep Convolutional Neural Network Based Medical Image Classification for Disease Diagnosis." Journal of Big Data 6 (1): 113. https://doi.org/10.1186/s40537-019-0276-2.

\section{Special Thanks}

The authors would like to thank Connor Esterwood for referring the author to valuable iconography as well as Chris Kerr for the Noun Project mobile phone icon in Figure 2. 